\title{
The impact of sample processing and media chemistry on the culturable diversity of bacteria isolated from a cave
}

\author{
Katey E. Bender (1)", Katelyn Glover, Alexander Archey \& Hazel A. Barton (1) \\ Department of Biology, University of Akron, 302 Buchtel Common, Akron, OH 44325, USA
}

\begin{abstract}
Although molecular approaches can identify members of microbial communities in the environment, genomic information does not necessarily correlate with environmental phenotype. Understanding functional roles can be done by cultivating representative species, yet the culturablility of bacteria from caves remains low, at $0.02 \%$, limiting our understanding of microbial community interactions and processes. We have investigated several factors influencing culturability of bacteria from a single sample location in Maxwelton Sink Cave, WV, USA. Extended incubation of inoculated plates showed a significant increase in colony counts from two to four weeks, indicating that extended incubations increase culturability. There were no significant differences in plate counts or diversity measures when the sample was suspended in different buffers prior to cultivation, while samples plated immediately after collection demonstrated higher culturability. Although supplementing the media with antibiotics reduced colony counts and cultured diversity, these plates did appear to contain a higher proportion of slow-growing oligotrophs. Finally, among a selection of culture media used, pyruvate agar showed the highest culturability and bacterial diversity, which may be a result of the oxygen radical scavenging effects of pyruvate. By identifying methods that improve culturable diversity, we hope to further understand the roles played by bacteria in cave communities, and test hypotheses that are best assessed using culture-based methods, such as screening for bioactive compounds or confirming in situ metabolic strategies.
\end{abstract}

Keywords: bacteria, cave, cultivation, culturable diversity, culturomics

Received 24 June 2020; Revised 21 October 2020; Accepted 21 October 2020

Citation: Bender K.E., Glover K., Archey A. and Barton H.A., 2020. The impact of sample processing and media chemistry on the culturable diversity of bacteria isolated from a cave. International Journal of Speleology, 49 (3), 209-220. Tampa, FL (USA) ISSN 0392-6672

https://doi.org/10.5038/1827-806X.49.3.2337

\section{INTRODUCTION}

Caves contain diverse microbial populations, despite limitations in energy input (Barton, 2015). The majority of this diversity has been revealed through molecular approaches, which have identified both a core biome and rarer specialists (Hershey \& Barton, 2018). Although DNA sequencing and metagenomics can reveal bacterial metabolic potential, cultivation gives a better idea of functional metabolic activity under different conditions. Nonetheless, our ability to culture microorganisms from the environment is limited. This is the so-called "great plate count anomaly", which describes the discrepancy between the total number of bacterial cells in an environmental sample (which may be counted using microscopic techniques) and the culturable population of that sample (Winterberg, 1898; Staley \& Konopka, 1985). It has been estimated that for most environments, only $0.1-1 \%$ of species can be cultured under standard laboratory conditions (Amann et al., 1995; Kaeberlein et al., 2002; Vartoukian et al., 2010). This "culturable fraction" is even lower in caves, where previous work has seen culturability as low as $0.02 \%$ of the total cells present (Barton et al., 2006). The low culturability of cave microbes therefore presents a significant barrier to understanding metabolic activities that support microbial growth in this environment.

Strategies for increasing cultivation have been explored in soil and marine environments (Vartoukian et al., 2010; Stewart, 2012; Overmann et al., 2017). A recent increase in the cultivation of targeted organisms is linked to identifying the necessary nutritional requirements by genome sequencing and key metabolic requirements (e.g., Könneke et al., 2005; Tripp et al., 2008; Bomar et al., 2011; Overmann et al., 2017). This strategy has also been employed in an untargeted manner, where synthetic growth media are designed to replicate the environmental conditions, for example through mineral composition, sources of 
organic carbon, and pH (e.g., Zengler, 2009; Stewart, 2012). These are a number of strategies that have yet to be comparatively explored in cave culturability:

1) Extended incubation times have been shown to increase culturable diversity (Zengler, 2009; Vartoukian et al., 2010); this strategy contributed to the first isolation of the SAR 11 and OM43 clades (Connon \& Giovannoni, 2002; Rappé et al., 2002), and is key in culturing extreme oligotrophs (Song et al., 2009). Despite conflicting results on whether extended incubations increase the likelihood of cultivating other rare species (Davis et al., 2005; Kurm et al., 2019), prolonged incubation does appear to improve diversity for slow-growing oligotrophs (Alain \& Querellou, 2009).

2) Reducing organic carbon inputs in culture media to approach the level of carbon in caves, which commonly have organic carbon inputs of less than $0.5 \mathrm{mg} / \mathrm{L}$ (Barton, 2015; Hershey \& Barton, 2018), can also be an effective cultivation strategy. Conventional culture media (e.g., tryptic soy broth or R2A) contain organic carbon several orders of magnitude higher than that in caves and other oligotrophic environments (Rappé et al., 2002; Overmann et al., 2017). As a result, such media must be diluted before use (e.g., Watve, Shejval, Sonawane, Rahalkar, Matapurkar, et al., 2000; Aagot et al., 2001; Connon \& Giovannoni, 2002; Janssen et al., 2002), which has been a successful strategy for culturing oligotrophic taxa (e.g., SAR11, Rappé et al., 2002; Verrucomicrobia, Sangwan et al., 2005). Alternately, using culture media with a range of organic carbon sources and mineral compositions can also increase the diversity of cultured isolates from an environment (e.g., Köpke et al., 2005; Vartoukian et al., 2010; Kurm et al., 2019).

3) Slower-growing species that are underrepresented in culture libraries may be selected for by supplementing culture media with low concentrations of antibiotics (e.g., chloramphenicol and nalidixic acid). Theoretically, these antibiotics would slow the growth of fast-growing culture "weeds": $r$-strategists, opportunistic fast growing species with flexible metabolic strategies. In contrast, the slower-growing oligotrophs (that are less represented in culture libraries) are $K$-strategists, whose growth rates are fixed but are better adapted to low-nutrient conditions (Andrews \& Harris, 1986; Watve, Shejval, Sonawane, Rahalkar, Matapurkar, et al., 2000; Zengler, 2009; Barton, 2015). In high-nutrient conditions, r-strategists should out-compete $K$-strategists; by including antibiotics, the growth of these organisms should be slowed and/or inhibited in favour of cultivating a greater diversity of oligotrophs (Alain \& Querellou, 2009).

4) The buffer in which solid samples are resuspended may also influence culturability. Previous studies of cave communities (e.g., Bhullar et al., 2012) have resuspended samples in sterilized water obtained from that cave. Although this may improve culturability by making sample suspensions geochemically similar to the cave environment (e.g., Zengler, 2009; Stewart, 2012), osmotic stress and low salinity have been shown to induce a viable but non culturable state in bacteria (Gin \& Goh, 2013; Li et al., 2014; Dong et al., 2020). This suggests that suspension solutions buffered to physiological conditions (for example, pH, sodium, and potassium; Kobayashi, 1985; Padan et al., 2005) may be effective for increasing culturability.

Finally, caves include additional challenges for cultivation-based research: travelling to sample collection sites may require negotiating vertical obstacles and/or small spaces in the cave, along with arduous travel times from the entrance. Research materials must also be transported by hand, requiring an approach that is efficient in the weight and volume of materials transported into the cave when sampling the most remote sites. One way of overcoming these limitations is by collecting samples in the cave, but returning to the entrance for sample processing and plating, which may not occur until several hours later.

It is unknown how each of these approaches (varying carbon source, nutrient load, antibiotics, and time between sample collection and processing) influences bacterial culturability from caves. In order to examine each of these, we carried out an extensive culture trial in Maxwelton Sink Cave, West Virginia, a relatively shallow, epigenic cave (145 $\mathrm{m}$ depth), with an upper level that is easily accessible for sampling and a gate to limit access (West Virginia Cave Conservancy, 2018; N. Socky, pers. comm., 2020). Maxwelton Sink Cave is therefore a broadly representative cave environment to examine the influence of each of these approaches on culturability, which are assessed using direct cell counting and sequence-based methodologies.

\section{METHODS}

\section{Sample collection and culture conditions}

We decided to test the impact of a number of parameters on culturability of samples. These included: sample suspension buffer; processing time (immediately on site, or post-collection); the addition of antibiotics; and carbon-chemistry of the cultivation media. We collected eight individual $\sim 5 \mathrm{~g}$ pieces of rock from the same wall location in Maxwelton Sink Cave, which were crushed using a sterile mortar and pestle then suspended in liquid for further processing. Sample processing parameters are summarized in Table 1.

One rock portion (CC) was crushed on site immediately after collection and preserved in filtered $(0.2 \mu \mathrm{m}) 70 \%$ ethanol for cell counting. The control rock (CTL) was crushed on site, resuspended in 10 $\mathrm{mL} 0.2 \mu \mathrm{m}$ filter-sterilized cave water, and inoculated (0.1 mL) on diluted R2A agar (1:100 and 1:1,000; BD Difco, Franklin Lakes, NJ) and water agar (containing $1.5 \%$ agar only). In order to limit the growth of fast growing bacterial species, $10 \mu \mathrm{g} / \mathrm{mL}$ nalidixic acid and chloramphenicol (Sigma-Aldrich, St. Louis, MO) were added, while $4 \mu \mathrm{g} / \mathrm{mL}$ nystatin (Fisher Scientific, 
Table 1. Sample collection parameters for each group of subsamples. Standard media consists of water agar, 1:1,000 R2A agar, and 1:100 R2A agar.

\begin{tabular}{|c|c|c|c|c|c|}
\hline ID & Treatment & Processing & Buffer & Media & Antibiotics \\
\hline $\mathrm{CC}$ & Cell Counting & - & - & - & - \\
\hline CTL & Control & Immediately & Cave water & Standard media & Included \\
\hline $\mathrm{BP}$ & \multirow{2}{*}{ Buffers } & \multirow{2}{*}{ Immediately } & PBS & \multirow{2}{*}{ Standard media } & \multirow{2}{*}{ Included } \\
\hline BR & & & Ringers & & \\
\hline $\mathrm{PL}$ & \multirow{2}{*}{ Processing } & Crushed on site, plated $6 \mathrm{~h}$ later & \multirow{2}{*}{ Cave water } & \multirow{2}{*}{ Standard media } & \multirow{2}{*}{ Included } \\
\hline $\mathrm{CL}$ & & Crushed and plated $6 \mathrm{~h}$ later & & & \\
\hline $\mathrm{nAB}$ & Antibiotics & Immediately & Water & Standard media & Not included \\
\hline OM-SA & \multirow{6}{*}{ Other Media } & \multirow{6}{*}{ Immediately } & \multirow{6}{*}{ Cave water } & Soil agar & \multirow{6}{*}{ Included } \\
\hline OM-PA & & & & Pyruvate agar & \\
\hline OM-ISP2 & & & & ISP2 & \\
\hline OM-IS4 & & & & ISP4 & \\
\hline OM-ISP5 & & & & ISP5 & \\
\hline OM-ISP7 & & & & ISP7 & \\
\hline
\end{tabular}

Waltham, MA) was included to limit fungal growth.

Buffers used included filter-sterilized cave water, Ringer's solution (2.25 g NaCl, $0.05 \mathrm{~g} \mathrm{NaHCO}_{3}, 0.12 \mathrm{~g}$ $\mathrm{CaCO}_{3}$ ), and phosphate-buffered saline (PBS; SigmaAldrich, St. Louis, MO). Rock portions were either crushed on site and streaked on media immediately, crushed on site and inoculated onto media $\sim 6$ hours later, or crushed $\sim 6$ hours after collection and then inoculated onto media. The role of antibiotics was evaluated by plating identical media with and without the addition of chloramphenicol and nalidixic acid (lack of antibiotics is indicated by nAB). Alternate carbon sources in media included pyruvate agar (see Barton et al., 2005), soil agar (see Nguyen et al., 2018), and ISP media 2, 4, 5, and 7 from the International Streptomyces project (see Shirling \& Gottlieb, 1966). All crushed rock portions were resuspended in $10 \mathrm{~mL}$ of the appropriate buffer for each set of sampling conditions (Table 1) and a 0.1 $\mathrm{mL}$ volume was inoculated onto plates using a sterile swab. Resuspended rock for all sets of sampling conditions were inoculated onto agar in triplicate, transported back to the lab at $4^{\circ} \mathrm{C}$ and then incubated in the dark at $25^{\circ} \mathrm{C}$ for 4 weeks. Although $4 \mu \mathrm{g} / \mathrm{mL}$ nystatin was added to all media to limit fungal growth, some plates did demonstrate fungal contamination, presumably from nystatin-resistant genera. Plates with fungal growth were discarded from additional analyses.

\section{Cell and Colony Forming Unit (CFU) counting}

To determine the total number of cells present in the sample, $0.1 \mathrm{~mL}$ of the rock preserved in $70 \%$ ethanol (CC) was added to $0.9 \mathrm{~mL}$ sterile PBS and stained with SYBR Green I for 15 minutes, following the manufacturer's recommended protocol (SigmaAldrich, St. Louis, MO). The full stained sample was filtered onto a $25 \mathrm{~mm}$ diameter, $0.2 \mu \mathrm{m}$ membrane filter (Anodisc or Cyclopore; Whatman, Piscataway, $\mathrm{NJ}$ ) and counted at a $1,000 \mathrm{x}$ magnification via epifluorescence using an Olympus BX53 microscope (Olympus America Inc, Center Valley, PA). The average number of cells from at least 50 fields-of-view was used to calculate the number of cells per $\mathrm{mL}$ in the original suspension (Hershey et al., 2018). Visible colonies on plates were counted every two weeks.
To determine how these colony counts compared to the total number of cells in the original rock sample, triplicate subsamples of $1 \mathrm{~mL}$ of each suspended, crushed portion of rock were dried and weighed. This allowed us to determine the average weight (in g) of rock per $\mathrm{mL}$, and hence the average amount of material in the $0.1 \mathrm{~mL}$ streaked on plates or used in microscopy. Using these values, direct cell counts and CFUs could be standardized per g of the rock sample. Percent culturability was calculated by dividing plate count values (per g) the number of cells counted via microscopy (per g).

\section{Molecular techniques and DNA sequencing}

Colonies were collected from the surface of the plate using $5 \mathrm{~mL}$ of the buffer in which the crushed rock portion was originally suspended. Of this, $2 \mathrm{~mL}$ was centrifuged in an Eppendorf 5424 microcentrifuge (Eppendorf AG, Hamburg, Germany) at maximum speed $(21,130 \times \mathrm{g})$ for two minutes to form a pellet for genomic DNA extraction using a Zymo QuickDNA Fungal/Bacterial Miniprep Kit (Zymo Research, Irvine, CA). The 16S rRNA gene sequences were then amplified via polymerase chain reaction (PCR) using a modified version of the protocol outlined in Caporaso, Lauber, Walters, Berg-Lyons, Huntley, et al. (2012). Briefly, Illumina iTag-barcoded universal primers targeting the V4 region of the bacterial/archaeal 16S rRNA gene $(515 \mathrm{~F}$ 5'-GTGYCAGCMGCCGCGGTAA; 806R 5'-GGACTACNVGGGTWTCTAAT) were used with Illumina multiplexed (barcoded) sequencing (Apprill et al., 2015; Parada et al., 2016). Amplification was carried out using HotStarTaq Plus Master Mix Kit (Qiagen, Germantown, MD) and an initial denaturing step at $94^{\circ} \mathrm{C}$ for 3 minutes, followed by 30 cycles of $94^{\circ} \mathrm{C}$ for $45 \mathrm{~s}, 53^{\circ} \mathrm{C}$ for $40 \mathrm{~s}$, and $72^{\circ} \mathrm{C}$ for $60 \mathrm{~s}$, and a final elongation step at $72^{\circ} \mathrm{C}$ for $5 \mathrm{~min}$. The PCR products were checked for successful amplification using gel electrophoresis, pooled, and purified using Ampure XP beads. Sequencing was carried out using the Illumina MiSeq platform $(2 \times 250)$ by MrDNA Molecular Research (Shallowater, TX).

Sample diversity was determined from the Illumina data using QIIME version 1.9.1 (Caporaso, Kuczynski, Stombaugh, Bittinger, Bushman, et al., 2010). Paired end reads were joined and barcodes extracted, then 
iTag libraries were demultiplexed and merged into a complete dataset for each sample. Chimera checking was performed, followed by open-reference OTU picking (Haas, Gevers, Earl, Feldgarden, Ward, et al., 2011; Caporaso, Lauber, Walters, Berg-Lyons, Huntley, et al., 2012; Rideout, He, Navas-Molina, Walters, Ursell, et al., 2014). The OTUs generated were assigned using the SILVA QIIME 16S reference database (SILVA 132; Quast et al., 2013).

Subsequent analyses of diversity were performed using QIIME and R ( $\mathrm{R}$ version 3.6.1, R Core Team, 2019). The phyloseq package in $R$ (McMurdie \& Holmes, 2013) was used to determine alpha-diversity, using observed OTUs and inverse Simpson metrics (Simpson, 1949). The phyloseq package was also used to generate principal coordinate analysis (PCoA) plots using the unweighted UniFrac metric to visualize differences in community structure (Lozupone \& Knight, 2005). phyloseq analyses were visualized using the ggplot2 package (Wickham, 2016).

\section{RESULTS}

The culturability of bacteria in Maxwelton Sink Cave was measured in two ways: the total number of culturable isolates, and the diversity of these isolates. We used direct cell counting via fluorescence microscopy to determine the total number cells in the rock sample, which was $1.75 \times 10^{6}$ cells/g (SD $\pm 45.3 \%)$. Culturability was measured by counting colonies at two and four weeks after initial plating. Across all tested conditions, there was a statistically significant increase in observable colony counts (Student's t-test, $p=0.001$ ), with average of $4.63 \mathrm{x}$ $10^{3} \mathrm{CFU} / \mathrm{g}$ after two weeks, increasing to $5.97 \times 10^{3}$ $\mathrm{CFU} / \mathrm{g}$ at four weeks, demonstrating a 1.5 to 2 -fold increase in culturability.

Culturability, expressed as the percentage of culturable cells from the rock sample, was calculated for each growth condition (Table 2). When using media that had previously been using in caves (water and dilute R2A agar) the greatest culturability was observed when no nutrients were added to the media (water agar; Table 2). Media with varying carbon sources showed mixed results, from little to no growth, such as ISP2, ISP4 and soil agar with no growth, $0.006 \%$ and $0.19 \%$ respectively, to pyruvate, which demonstrated $1.76 \%$ culturability. The growth on pyruvate was higher than any other media used
(Table 2). Our data also demonstrated that the absence of antibiotics increased culturability.

Our cultivation approaches resulted in individual media plates with growth ranging from none, to hundreds of colonies. The colonies were washed off of all the plates for analysis. While we obtained sequence data for plates with low colony counts $(<10 \mathrm{CFU} /$ plate), the Illumina data obtained for these plates had a low number of sequence reads and those below an average of 75,000 reads/sample were excluded from further analysis (Fig. 1; yellow points). Other samples were eliminated based on a high $(>0.5 \%)$ presence of Euryarchaeota or unassigned OTUs, which would not be expected to be present in cultivation-based datasets, suggesting contamination in the sequence data (Fig. 1, green points; Solden et al., 2016; Baker et al., 2020). Poorly-represented taxa $(<0.1 \%$ relative family-level abundance in a sample) were excluded from further analysis in their respective samples. The removal of these samples made direct comparison of community structure difficult, as we often had only one available sample per condition, so we focused our efforts on understanding broader measures of diversity.

\section{Role of sample suspension buffer in culturability}

To assess the impact of resuspension buffer on culturability, we used Ringer's solution, PBS, and sterile cave water $(\mathrm{pH} 8.2)$ to resuspend rock samples before inoculation onto plates. The results (Fig. 2A) demonstrate that the rock samples suspended in water produced the greatest number of colonies when plated on water agar. This trend was not repeated for other media. For example, on R2A agar, PBS increased colony counts compared with the other buffers. These data suggest that sample suspension buffers may impact culturability depending on the growth medium, although significance was not seen for the impact of buffer alone (Single-factor ANOVA, $\mathrm{F}_{2,24}=1.22, p=0.314$ ) or any combination of media and buffer (Two-factor ANOVA, $\mathrm{F}_{4,18}=1.09, p=0.392$ ). In order to determine if there were any similarities between community diversity based on suspension buffer, we used a principal coordinate analysis (PCoA) to evaluate beta-diversity, which incorporates OTU phylogenetic distance into community comparisons. The PCoA plot in Fig. 2B demonstrates a high degree of overlap in diversity between communities, suggesting that there are no meaningful differences in culturable diversity when using different buffers.

Table 2. Average culturability by sample collection condition.

\begin{tabular}{|c|c|c|c|c|c|c|c|}
\hline Media & CTL & BP & BR & PL & CL & $\mathbf{n A B}$ & OM \\
\hline Water agar & $0.970 \%$ & $0.130 \%$ & $0.205 \%$ & $0.294 \%$ & $0.051 \%$ & $1.307 \%$ & - \\
\hline $1: 1,000 \mathrm{R} 2 \mathrm{~A}$ & $0.133 \%$ & $0.225 \%$ & $0.058 \%$ & $0.204 \%$ & $0.022 \%$ & $0.797 \%$ & - \\
\hline 1:100 R2A & $0.261 \%$ & $0.347 \%$ & $0.101 \%$ & $0.025 \%$ & $0.022 \%$ & $1.143 \%$ & - \\
\hline Pyruvate agar & - & - & - & - & - & - & $1.759 \%$ \\
\hline Soil agar & - & - & - & - & - & - & $0.019 \%$ \\
\hline ISP2 & - & - & - & - & - & - & $0.000 \%$ \\
\hline ISP4 & - & - & - & - & - & - & $0.006 \%$ \\
\hline ISP5 & - & - & - & - & - & - & $0.094 \%$ \\
\hline ISP7 & - & - & - & - & - & - & $0.003 \%$ \\
\hline
\end{tabular}




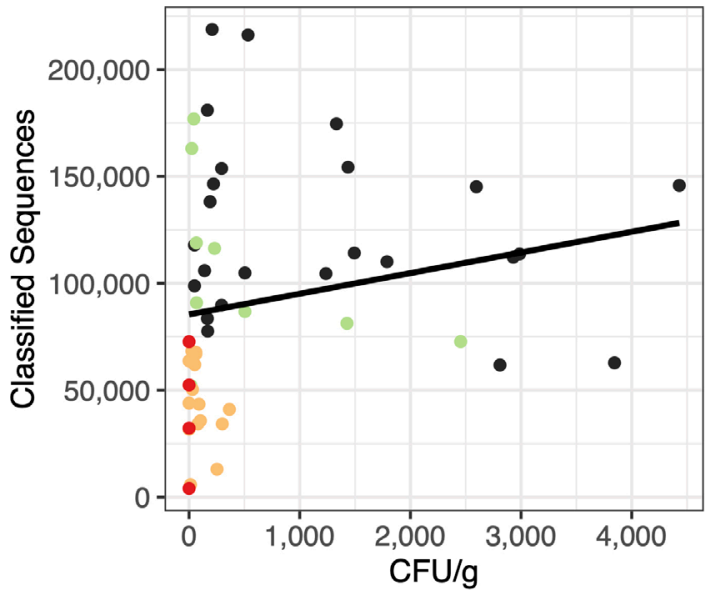

Illumina Sequences

- Included in analysis

- Excluded by sequence quality

Excluded by taxonomy

- Blank controls

Fig. 1. Plotted relationship between the number of sequences reads for DNA extracted from each plate, compared with the observed number of CFU on the same plate. The calculated $R^{2}=0.0419$ was low and indicated significant noise in the sequencing data at lower CFU counts ( $500 \mathrm{CFU} / \mathrm{g}$ is equivalent to less than $10 \mathrm{CFU} /$ plate).
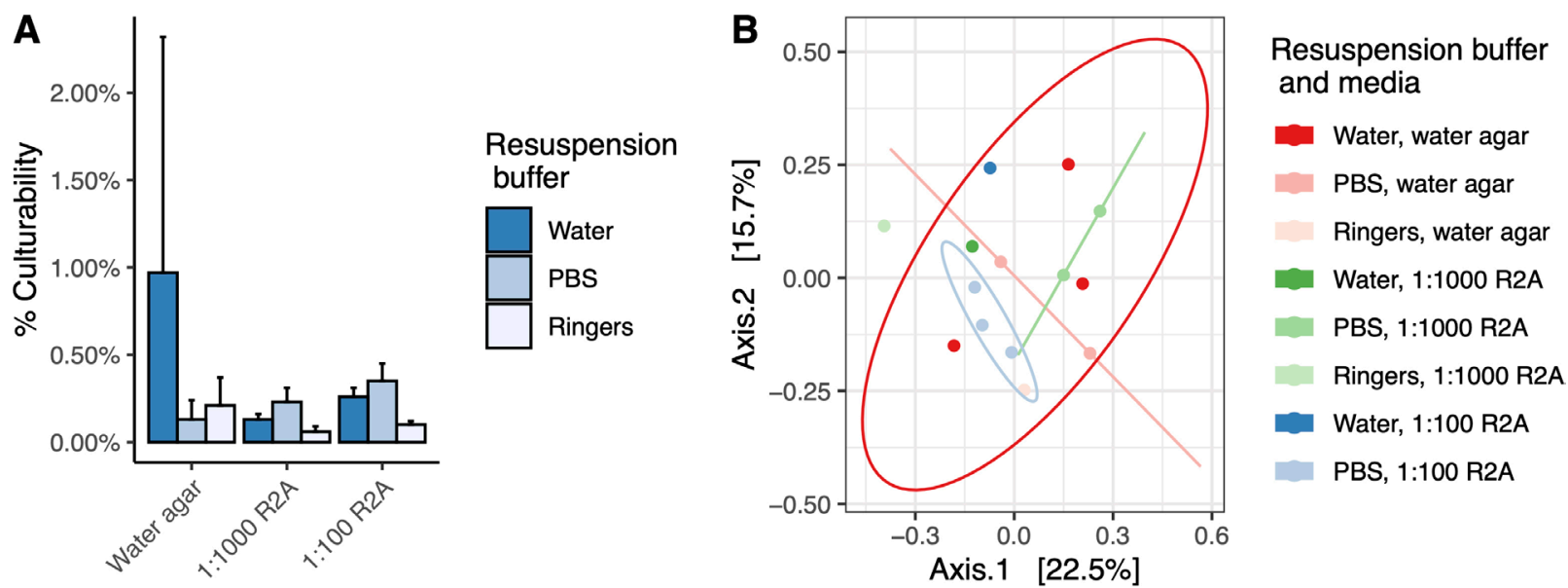

Fig. 2. The influence of suspension buffer on bacterial culturability (A) (based on colony forming units per g of crushed rock) and beta-diversity (B) based on Illumina sequencing of growth on plates. Beta-diversity is represented by a PCoA of the unweighted UniFrac metric; ellipses represent $95 \%$ confidence intervals.

\section{Role of sample processing in culturability}

To evaluate the impact of sample processing time (i.e., the time when the rock sample was crushed and plated onto media after collection) on culturability, we examined rock samples that were crushed and plated immediately after collection, crushed immediately and plated $\sim 6$ hours later, and crushed and plated $\sim 6$ hours later (Fig. 3). Rock samples plated on water agar and 1:100 R2A agar suggest that rock portions crushed on-site and inoculated immediately demonstrate the highest culturability; however, this trend was not statistically significant (ANOVA, $\left.\mathrm{F}_{2,24}=1.92, p=0.169\right)$. Only rock samples processed immediately on site generated enough colonies for high-quality sequence data analysis. The rock samples where processing was delayed produced so few colonies that they were eliminated in the sample curation process (see Fig. 1). As a result, no taxonomic or diversity analyses can be performed for this dataset.

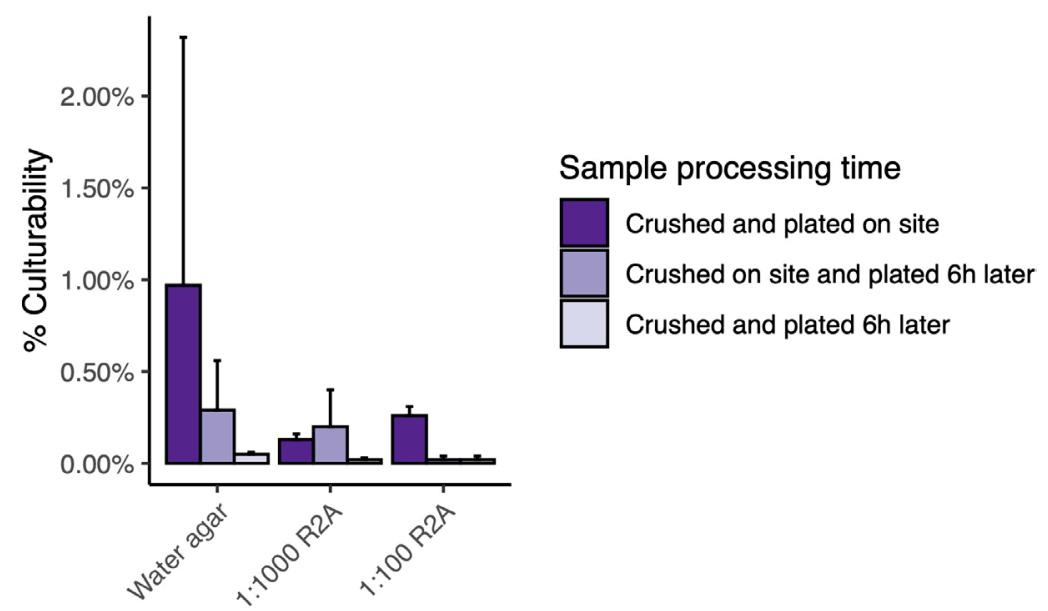

Fig. 3. The influence of processing time on bacterial culturability (based on colony forming units per $g$ of crushed rock) of samples after collection. 


\section{Role of antibiotics in culturable diversity}

To assess the effect of antibiotics on culturability, media were prepared with and without $10 \mu \mathrm{g} /$ $\mathrm{mL}$ chloramphenicol and nalidixic acid. At low concentrations, these antibiotics are bacteriostatic (i.e., they inhibit growth, but do not kill bacteria), inhibiting translation and DNA replication, respectively (Jardetzky, 1963; Emmerson \& Jones, 2003). As a result, these antibiotics have previously been used to inhibit the growth of rapidly growing species in culture that would otherwise overwhelm the plate and reduce overall diversity. The data (Fig. 4A) suggest that antibiotics suppress overall bacterial growth, and media without antibiotics had a statistically significant increase in colony counts (ANOVA, $\mathrm{F}_{1,16}=4.60, p=0.0476$ ). In all cases, our data suggest that media without antibiotics contained both higher species richness and evenness in observed bacterial growth (Fig. 4B). Communities grown on water agar demonstrated a significant increase in both species richness and evenness, regardless of whether antibiotics were present (ANOVA; observed OTUs, $F_{1,4}=25.4, p=0.00727$; inverse Simpson, $\left.\mathrm{F}_{1,4}=15.2, p=0.0176\right)$. Nonetheless, the community structure (Fig. 4C) suggests that the actual diversity of isolates is distinct in the presence and absence of antibiotics. As we obtained sufficient Illumina data for samples on water agar, 1:1,000 R2A, and 1:100 R2A in both the presence and absence of antibiotics, we were able to carry out a comparative analysis of the bacterial diversity on these plates (Fig. 4D). The data support the PCoA analyses, demonstrating several distinctive differences between growth in the presence and absence of antibiotics. At the family level, plates without antibiotics were dominated by fast-growing members of the Actinobacteria (Dietziaceae, Dermacoccaceae, and Micrococcaceae) and Gammaproteobacteria (Burkholderiaceae and Moraxellaceae). In contrast, the use of antibiotics appears to select for the growth of Alphaproteobacteria, with higher relative abundances of members of the slow-growing Xanthobacteraceae and Reyranellaceae, and an increased prevalence of the Nocardiaceae and Promicromonosporaceae within the Actinobacteria. It is interesting to note that many of the differences in culturability with and without antibiotics were not always shared across media types. For example, antibiotics only enhanced the growth of the Intrasporangiaceae on water agar (12.6\% total growth), with little $(0.12 \%)$ or no representation in culture in culture on $1: 1,000$ and $1: 100 \mathrm{R} 2 \mathrm{~A}$, respectively. In contrast, media without antibiotics promoted growth of the Intrasporangiaceae, only slightly on water agar $(6.8 \%)$, but significantly on $1: 1,000$ and $1: 100$ R2A (15.4\% and $16.6 \%$, respectively). This suggests that the effects of nutrients within the media can differentially select for growth, depending upon other stressors on the cell.

\section{Impact of carbon source}

Finally, we examined the role of carbon source on culturability, using pyruvate, soil extract, and ISP2, ISP4, ISP5, and ISP7 media. The results (Fig. 5A) show that pyruvate agar produced the greatest culturability of any media used, followed by water agar. No significant improvement in culturability over water agar was seen on any other media. Soil agar and ISP2 were particularly poor in culturing isolates from this environment, with $0.003 \%$ and no growth, respectively. Although an ANOVA detected significant differences in culturability between all media types (ANOVA, $\mathrm{F}_{8,18}=5.04, p=0.002$ ), no significant difference was detected between pyruvate and previously used cave cultivation media (i.e., water agar, 1:1,000 R2A, and 1:100 R2A; ANOVA, F $=3.40, p=0.074)$. Illumina data suggested that the pyruvate agar produces a greater diversity of bacterial species than other media, although when compared with water agar and dilute R2A, these differences were not significant. The beta-diversity of these samples, visualized using PCoA (Fig. 5B) indicate significant differences in community structure between pyruvate agar and previously used cave culture media, suggesting that these media grow different subsets of the culturable species from the cave environment; however, we did not obtain sufficient data for a direct phylogenetic comparison.

\section{DISCUSSION}

Increasing the culturable diversity of microbes allows a more thorough assessment of the metabolic, biosynthetic, and functional activities of these organisms in their environment (Lagier et al., 2018). Increasing this culturability (culturomics) has been shown to dramatically increase our understanding of ecosystem function (Lagier et al., 2018). While previous work has indicated that caves are a potential source of novel natural products, including novel antibiotics (e.g., Laiz et al., 1999; Laiz et al., 2003; Bhullar et al., 2012; Leo et al., 2012; Cheeptham, 2013; Pawlowski et al., 2016; Adam et al., 2018), there have been no systematic studies on how to improve culturability from these environments. In this study, a number of sample processing conditions and growth media were assessed for their effectiveness in increasing culturable bacterial diversity from a single sample site. We hypothesized that factors in sample collection and incubation, such as the of physiological buffers to resuspend samples, processing samples immediately after collection, and using an extended (i.e., 4-week) incubation period could increase culturability. We also investigated the influence of the growth media with and without the addition of antibiotics to reduce fast growing species on increased culturable diversity. Using a single sample site to inoculate all of these culture conditions makes such comparative analyses possible.

We found a significant increase in culturability after extended incubation of the plates (from two to four weeks). This is in line with previous studies which have suggested that the number of cultured isolates increases with incubation time; however, there are conflicting results on whether these extended incubations increase the diversity of isolates or increase the likelihood of culturing rare species (Davis et al., 2005; Kurm et al., 2019). Because of the 

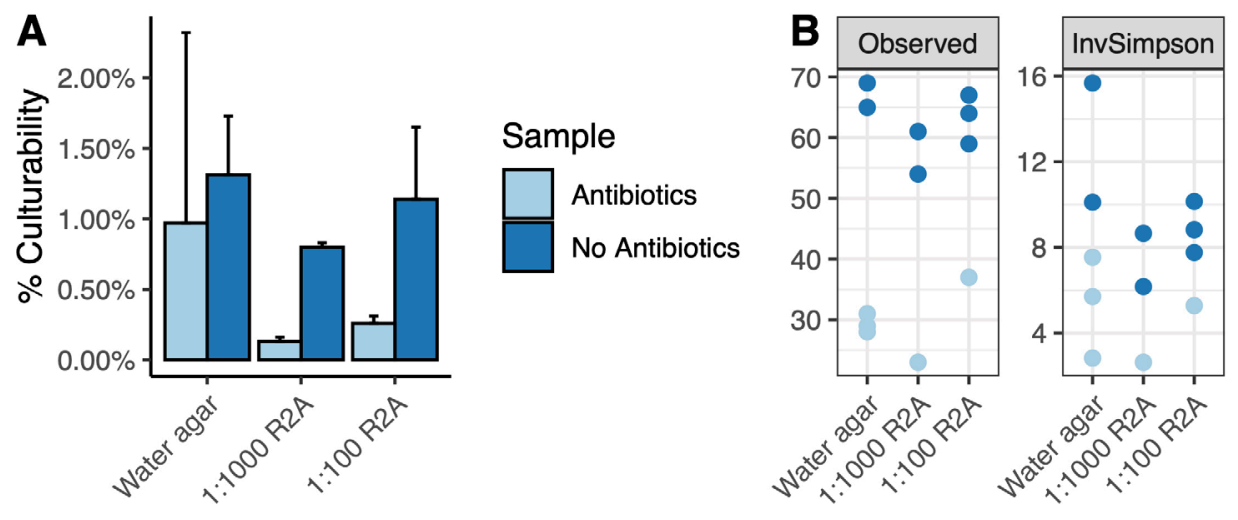

\section{Sample}

- Antibiotics

- No Antibiotics

C

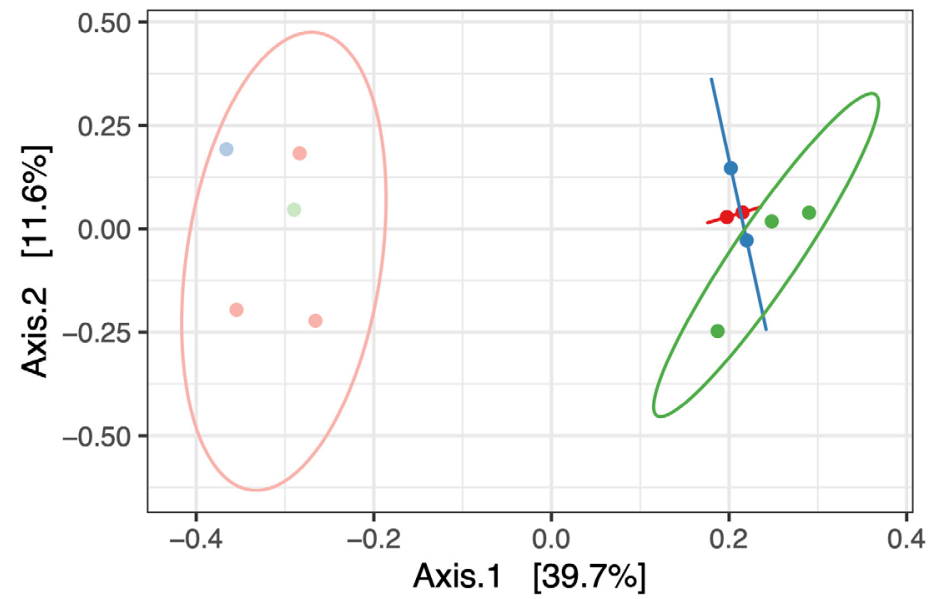

\section{Sample}

Antibiotics, water agar

Lu Antibiotics, water agar

Antibiotics, 1:1000 R2A

No Antibiotics, 1:1000 R2A

Antibiotics, 1:100 R2A

No Antibiotics, 1:100 R2A

D

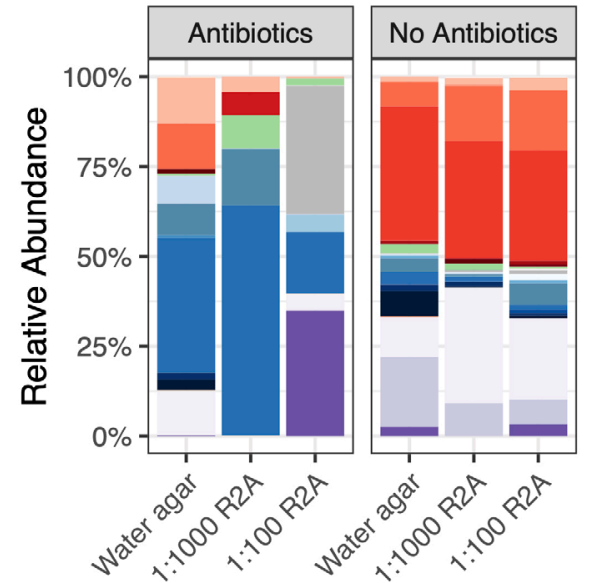

Family

Dietziaceae

Mycobacteriaceae

Nocardiaceae

Dermacoccaceae

Intrasporangiaceae

Micrococcaceae

Promicromonosporaceae

Nocardioidaceae

Streptomycetaceae

Chitinophagaceae

Spirosomaceae

JG30-KF-CM45

Bacillaceae

Paenibacillaceae

Planococcaceae

Azospirillaceae

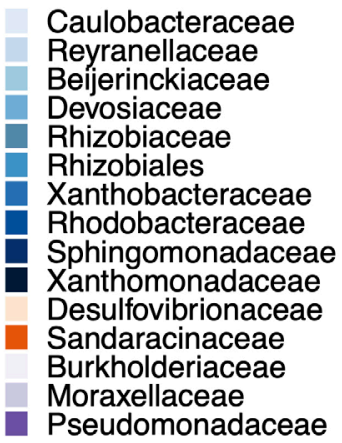

Caulobacteraceae Beijerinckiacea

Devosiaceae

Rhizobiaceae

Rhizobiales

Xanthobacteraceae

Rhodobacteraceae

Sphingomonadaceae

Xanthomonadaceae

Desulfovibrionacea

Burkholderiaceae

Pseudomonadaceae

Fig. 4. The influence of antibiotics on culturability (A) and diversity. Illumina sequencing of plate growth was used to obtain the alpha-diversity (B), assessed using observed OTUs (Observed) and the inverse Simpson (InvSimpson) indices; beta-diversity (C), represented by a PCoA of the unweighted UniFrac metric with ellipses representing $95 \%$ confidence intervals; and averaged taxonomic composition (D) of growth on media. Taxa are represented at the family level and grouped by colour at the order level.
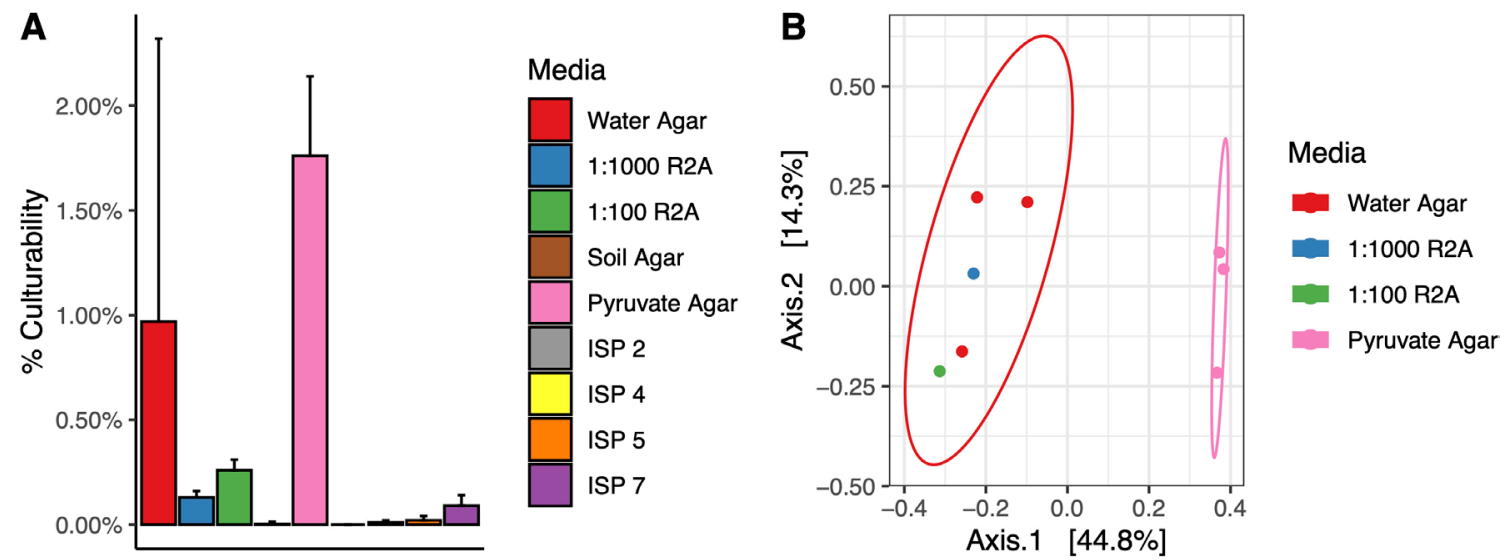

Fig. 5. The impact of different carbon sources in the media on culturability (A) and beta-diversity (B). Beta-diversity is obtained by Illumina sequencing of growth and is represented by a PCoA of the unweighted UniFrac metric; ellipses represent $95 \%$ confidence intervals. 
destructive sampling required for DNA extraction, we were only able to obtain sequence data from cultures with 4 weeks of growth and did not evaluate such changes in diversity, although such studies would prove important for future work.

Although the choice of sample suspension buffers showed no differences in culturability or diversity (Fig. 2), other factors did show pronounced effects, such as the use of antibiotics in the culture media. Past research on culturing cave microbes has included low concentrations of antibiotics (e.g., chloramphenicol and nalidixic acid) to suppress the growth of fastgrowing species ( $r$-strategists) in favour of slowergrowing oligotrophs ( $K$-strategists). Theoretically, in high-nutrient conditions, $r$-strategists should outcompete $K$-strategists and antibiotics should slow and/or inhibit fast-growing species in favour of cultivating a greater diversity of oligotrophs (Alain \& Querellou, 2009). Although alpha-diversity results (Fig. 4B) suggest that antibiotics may reduce the culturability of many bacterial species, the taxonomic distributions shown in Fig. 4D suggest that it does enrich for slow-growing species. The families that were more prevalent in media without antibiotics include the Micrococcaceae, Burkholderiaceae, and Moraxellaceae. Members of the Micrococcaceae and Burkholderiaceae are metabolically flexible (Coenye, 2014; Dastager et al., 2014), and the cultured Moraxellaceae belong to nonfastidious members of this bacterial family (Teixeira \& Merquior, 2014). All the families dominant in samples without antibiotics therefore have flexible metabolic strategies and higher growth rates - i.e., they are $r$-strategists.

In contrast, the families dominating the media containing antibiotics have slower growth rates and often benefit from strategies that reduce the proliferation of other bacteria when they are targeted for isolation. One of the most significant increases in relative abundance on media containing antibiotics is the family Xanthobacteraceae. While many of its member genera are often instead classified into the Bradyrhizobiaceae, both the Xanthobacteraceae and Bradyrhizobiaceae generally grow best on low nutrient media and their members commonly have slow growth rates (de Souza et al., 2014; Oren, 2014). The Reyranellaceae also show a considerable increase in relative abundance on media containing antibiotics. This is a poorly-cultured family with only five characterized species, which commonly require 2-7 days to grow sufficiently for analysis (Pagnier et al., 2011; Cui et al., 2017; Lee et al., 2017). Among the Actinobacteria, media containing antibiotics showed higher abundances of the Nocardiaceae and Promicromonosporaceae, both of which have been shown to benefit from targeted isolation strategies that limit the growth of other bacteria (Tiwari \& Gupta, 2012).

Together, these results suggest that antibiotics select against opportunistic, metabolically flexible $r$-strategists, and select for slower-growing $K$-strategists, as predicted. This is also supported by the community structures shown in Fig. $4 \mathrm{C}$, which indicate that media with and without antibiotics grow distinct species profiles. With the added context of the taxonomic results, we can infer that the opposing dominances of slow and fast-growing species in media with and without antibiotics are the major causes of these differences. These results suggest that to access the greatest culturable diversity of this environment, both media with and without antibiotics should both be used, even though media lacking antibiotics are likely to culture the fast-growing species that are already over-represented in culture libraries (Keller \& Zengler, 2004).

In contrast to previous cave work (Barton et al., 2005), our findings suggest that using pyruvate as a carbon source increases culturability, producing a distinctly different culturable profile when compared to other media (Fig. 5). In the previous work, the reduction in diversity on pyruvate agar was attributed to the ability of this organic carbon to feed directly into the tri-carboxylic acid cycle (Barton, 2015), although pyruvate does demonstrate oxygen radical scavenging properties. Other studies have shown that, when compounds inducing oxidative stress are present in culture media, there is a reduction in culturability and culturable diversity (Tanaka et al., 2014; Kawasaki \& Kamagata, 2017). This effect can be reversed, however, by including oxygen radical scavengers such as pyruvate or catalase in media, or by modifying media preparation protocols (Martin et al., 1976; Alain \& Querellou, 2009; Kato, Yamagishi, Daimon, Kawasaki, Tamaki, et al., 2018). These approaches may present a way to mitigate the metabolic stresses faced by caveadapted oligotrophs when inoculated on nutrient-rich culture media, orthogonal to the current strategy of using dilute culture media. Nonetheless, the increase in culturability seen must also be controlled against the use of HEPES buffer in the media, which has been shown to improve bacterial culturability in lake water bacteria (e.g., Bartscht et al., 1999, Bussmann et al., 2001).

In their study of soil bacteria, Janssen et al. (2002) established several techniques to increase culturability of soil communities. In that study, the authors increased their viable plate counts from $1.4 \%$ (using previously established techniques) to $14.1 \%$. Of the 30 isolates that they sequenced, 12 were members of then-uncultured taxa (Janssen et al., 2002). This result demonstrated that increasing culturability leads to an increase in rare species, making it reasonable to infer that increased $\mathrm{CFU}$ counts will result in increased diversity. Given the differences in resources required to assess culturability (colony counts) versus diversity (DNA extraction, PCR amplification and purification, Illumina sequencing, and bioinformatic analysis), colony counts may serve as a rough proxy to assess and optimize culture conditions (Lagier et al., 2018).

Based on the results we obtained, there are a number of possible avenues for future work investigating the culturability of cave microbial communities. Although over $80 \%$ of the colonies present in their final (12week) plate count were present after 4 weeks of incubation, Janssen et al. (2002) found that CFU counts continued to increase up to 10 weeks after 
inoculation; these later-growing colonies are more likely to be rare species (Davis et al., 2005; Kurm et al., 2019), indicating that extended incubations should be considered. Further studies should also attempt to more accurately replicate environmental conditions in both media and incubation conditions. This would be ideally achieved through the use of diffusion chambers (e.g., Nichols et al., 2010) for an initial, incave incubation followed by amending media (e.g., including carbonate compounds and buffering media to cave-relevant $\mathrm{pH}$, building on the results of Barton et al., 2005) and environmental conditions (e.g., light, temperature, humidity) to imitate the cave environment for improved isolation (Zengler, 2009; Stewart, 2012). Future work should also study the potential effects of different antibiotics in culture media on culturability, and a titration of antibiotic concentration to determine if there is an ideal combination/concentration to promote oligotrophic growth. To allow for comparative analyses, we used a single sample site for this study to reduce the impact of other variables, such as location (ceiling, floor, distance from entrance, depth) and nutrient availability on diversity. It is possible that the impacts of the cultivation techniques we have evaluated here may not translate to other cave surfaces, and comparative analyses from such sites would also be valuable.

Overall, these data suggest that the best strategy for cultivating the greatest diversity of bacteria in caves is to use a variety of culture media, as has been shown in other environments (e.g., Köpke et al., 2005; Alain \& Querellou, 2009; Vartoukian et al., 2010). This study has highlighted several culture techniques that influence the culturability of cave microbial communities, and suggests that there is considerable opportunity for culturomic approaches in cave environments.

\section{ACKNOWLEDGEMENTS}

The authors would like to thank the West Virginia Cave Conservancy for access to Maxwelton Sink Cave. Nick Socky is acknowledged for assistance in selecting an appropriate cave in which to perform this research and for navigational assistance; he and Rob Wardell are thanked for help carrying out field sampling. Thanks are also extended to Olivia Hershey for assistance and guidance carrying out analyses in QIIME and R, and to the two anonymous reviewers whose comments have improved the quality of this manuscript.

Authorship statement: KEB and $\mathrm{HAB}$ designed the study. KEB, KG, and HAB carried out sampling. KEB, $\mathrm{KG}$, and AA performed colony and cell counting. KEB extracted DNA and analyzed sequencing data. KEB wrote the manuscript with input from all authors.

\section{REFERENCES}

Aagot, N., Nybroe, O., Nielsen, P., Johnsen, K., 2001. An altered Pseudomonas diversity is recovered from soil by using nutrient-poor Pseudomonas-selective soil extract media. Applied and Environmental Microbiology, 67(11), 5233-5239.

https://doi.org/10.1128/AEM.67.11.5233-5239.2001

Adam, D., Maciejewska, M., Naômé, A., Martinet, L., Coppieters, W., Karim, L., Baurain, D., Rigali, S., 2018. Isolation, characterization, and antibacterial activity of hard-to-culture actinobacteria from cave moonmilk deposits. Antibiotics, 7(2), 28. https://doi.org/10.3390/antibiotics7020028

Alain, K., Querellou, J., 2009. Cultivating the uncultured: limits, advances and future challenges. Extremophiles, 13(4), 583-594.

https://doi.org/10.1007/s00792-009-0261-3

Amann, R.I., Ludwig, W., Schleifer, K.-H., 1995. Phylogenetic identification and in situ detection of individual microbial cells without cultivation. Microbiology and Molecular Biology Reviews, 59(1), 143-169. https://doi.org/10.1128/MMBR.59.1.143-169.1995

Andrews, J.H., Harris, R.F., 1986. r-and $K$-selection and microbial ecology. In: Marshall, C.K. (Ed.), Advances in Microbial Ecology. Springer Science + Business Media, New York, p. 99-147. https://doi.org/10.1007/978-1-4757-0611-6_3

Apprill, A., McNally, S., Parsons, R., Weber, L., 2015. Minor revision to V4 region SSU rRNA 806R gene primer greatly increases detection of SAR11 bacterioplankton. Aquatic Microbial Ecology, 75(2), 129-137. https://doi.org/10.3354/ame01753

Baker, B.J., De Anda, V., Seitz, K.W., Dombrowski, N., Santoro, A.E., Lloyd, K.G., 2020. Diversity, ecology and evolution of Archaea. Nature Microbiology, 5, 887-900. https://doi.org/10.1038/s41564-020-0715-Z

Barton, H., Taylor, N., Lubbers, B., Pemberton, A., 2006. DNA extraction from low-biomass carbonate rock: an improved method with reduced contamination and the low-biomass contaminant database. Journal of Microbiological Methods, 66(1), 21-31.

https://doi.org/10.1016/j.mimet.2005.10.005

Barton, H.A., 2015. Starving artists: bacterial oligotrophic heterotrophy in caves. In: Engel, A.S. (Ed.), Microbial life of cave systems, DeGruyter, Berlin, p. 79-104.

Barton, H.A., Pemberton, A., Millette, J., 2005. Comparative study of oligotrophic bacterial species cultivated from Jack Bradley Cave, Kentucky. In: Proceedings of the 14th International Congress of Speleology, Athens, 1, 245-249.

Bartscht, K., Cypionka, H., Overmann, J., 1999. Evaluation of cell activity and of methods for the cultivation of bacteria from a natural lake community. FEMS Microbiology Ecology, 28(3), 249-259. https://doi.org/10.1111/j.1574-6941.1999.tb00580.x

Bhullar, K., Waglechner, N., Pawlowski, A., Koteva, K., Banks, E.D., Johnston, M.D., Barton, H.A., Wright, G.D., 2012. Antibiotic resistance is prevalent in an isolated cave microbiome. PLoS One, 7(4), e34953. https://doi.org/10.1371/journal.pone.0034953

Bomar, L., Maltz, M., Colston, S., Graf, J., 2011. Directed culturing of microorganisms using metatranscriptomics. MBio, 2(2), e00012-11. https://doi.org/10.1128/mBio.00012-11

Bussmann, I., Philipp, B., Schink, B., 2001. Factors influencing the cultivability of lake water bacteria. Journal of Microbiological Methods, 47(1), 41-50. https://doi.org/10.1016/S0167-7012(01)00289-5

Caporaso, J.G., Kuczynski, J., Stombaugh, J., Bittinger, K., Bushman, F.D., Costello, E.K., Fierer, N., Pena, A.G., Goodrich, J.K., Gordon, J.I., et al., 2010. QIIME allows analysis of high-throughput community sequencing data. Nature Methods, 7, 335-336.

https://doi.org/10.1038/nmeth.f.303 
Caporaso, J.G., Lauber, C.L., Walters, W.A., Berg-Lyons, D., Huntley, J., Fierer, N., Owens, S.M., Betley, J., Fraser, L., Bauer, M., et al., 2012. Ultra-high-throughput microbial community analysis on the Illumina HiSeq and MiSeq platforms. The ISME Journal, 6, 1621-1624. https://doi.org/10.1038/ismej.2012.8

Cheeptham, N., 2013. Advances and challenges in studying cave microbial diversity. In: Cheeptham, $\mathrm{N}$. (Ed.), Cave microbiomes: A novel resource for drug discovery. Springer-Verlag, New York, p. 1-34. https://doi.org/10.1007/978-1-4614-5206-5 1

Coenye, T., 2014. The family Burkholderiaceae. In: Rosenberg, E., DeLong, E., Lory, S., Stackebrandt, E., Thompson, F. (Eds.), The Prokaryotes - Alphaproteobacteria and Betaproteobacteria. Springer, Berlin, p. 759-776.

Connon, S.A., Giovannoni, S.J., 2002. High-throughput methods for culturing microorganisms in very-lownutrient media yield diverse new marine isolates. Applied and Environmental Microbiology, 68(8), 3878-3885. https://doi.org/10.1128/AEM.68.8.3878-3885.2002

Cui, Y., Chun, S.J., Ko, S.R., Lee, H.G., Srivastava, A., Oh, H.M., Ahn, C.Y., 2017. Reyranella aquatilis sp. nov., an alphaproteobacterium isolated from a eutrophic lake. International Journal of Systematic and Evolutionary Microbiology, 67(9), 3496-3500. https://doi.org/10.1099/ijsem.0.002151

Dastager, S.G., Krishnamurthi, S., Rameshkumar, N., Dharne, M., 2014. The Family Micrococcaceae. In: Rosenberg, E., DeLong, E., Lory, S., Stackebrandt, E., Thompson, F. (Eds.), The Prokaryotes - Actinobacteria. Springer, Berlin, p. 455-498. https://doi.org/10.1007/978-3-642-30138-4_168

Davis, K.E., Joseph, S.J., Janssen, P.H., 2005. Effects of growth medium, inoculum size, and incubation time on culturability and isolation of soil bacteria. Applied and Environmental Microbiology, 71(2), 826-834. https://doi.org/10.1128/AEM.71.2.826-834.2005

de Souza, J.A.M., Carrareto Alves, L.M., de Mello Varani, A., de Macedo Lemos, E.G., 2014. The Family Bradyrhizobiaceae. In: Rosenberg, E., DeLong, E., Lory, S., Stackebrandt, E., Thompson, F. (Eds.), The Prokaryotes - Alphaproteobacteria and Betaproteobacteria. Springer, Berlin, p. 135-154. https://doi.org/10.1007/978-3-642-30197-1 253

Dong, K., Pan, H., Yang, D., Rao, L., Zhao, L., Wang, Y., Liao, X., 2020. Induction, detection, formation, and resuscitation of viable but non-culturable state microorganisms. Comprehensive Reviews in Food Science and Food Safety, 19(1), 149-183.

https://doi.org/10.1111/1541-4337.12513

Emmerson, A., Jones, A., 2003. The quinolones: decades of development and use. Journal of Antimicrobial Chemotherapy, 51(suppl. 1), 13-20. https://doi.org/10.1093/jac/dkg208

Gin, K.Y.-H., Goh, S.G., 2013. Modeling the effect of light and salinity on viable but non-culturable (VBNC) Enterococcus. Water Research, 47(10), 3315-3328. https://doi.org/10.1016/j.watres.2013.03.021

Haas, B.J., Gevers, D., Earl, A.M., Feldgarden, M., Ward, D.V., Giannoukos, G., Ciulla, D., Tabbaa, D., Highlander, S.K., Sodergren, E., et al., 2011. Chimeric 16 s rRNA sequence formation and detection in Sanger and 454-pyrosequenced PCR amplicons. Genome Research, 21(3), 494-504. https://doi.org/10.1101/gr.112730.110

Hershey, O.S., Barton, H.A. (2018). The microbial diversity of caves. In: Moldovan, O.T., Kováč, L., Halse, S. (Eds.), Cave ecology. Springer International Publishing, Basel, p. 69-90.

https://doi.org/10.1007/978-3-319-98852-8 5
Hershey, O.S., Kallmeyer, J., Wallace, A., Barton, M.D., Barton, H.A., 2018. High microbial diversity despite extremely low biomass in a deep karst aquifer. Frontiers in Microbiology, 9, 2823.

https://doi.org/10.3389/fmicb.2018.02823

Janssen, P.H., Yates, P.S., Grinton, B.E., Taylor, P.M., Sait, M., 2002. Improved culturability of soil bacteria and isolation in pure culture of novel members of the divisions Acidobacteria, Actinobacteria, Proteobacteria, and Verrucomicrobia. Applied and Environmental Microbiology, 68(5), 2391-2396.

https://doi.org/10.1128/AEM.68.5.2391-2396.2002

Jardetzky, O., 1963. Studies on the mechanism of action of chloramphenicol I. The conformation of chloramphenicol in solution. Journal of Biological Chemistry, 238(7), 2498-2508.

https://www.jbc.org/content/238/7/2498.short

Kaeberlein, T., Lewis, K., Epstein, S.S., 2002. Isolating "uncultivable" microorganisms in pure culture in a simulated natural environment. Science, 296, 11271129. https://doi.org/10.1126/science. 1070633

Kato, S., Yamagishi, A., Daimon, S., Kawasaki, K., Tamaki, H., Kitagawa, W., Abe, A., Tanaka, M., Sone, T., Asano, K., Kamagata, Y., 2018. Isolation of previously uncultured slow-growing bacteria by using a simple modification in the preparation of agar media. Applied and Environmental Microbiology, 84(19), e00807-18. https://doi.org/10.1128/AEM.00807-18

Kawasaki, K., Kamagata, Y., 2017. Phosphate-catalyzed hydrogen peroxide formation from agar, gellan, and k-carrageenan and recovery of microbial cultivability via catalase and pyruvate. Applied and Environmental Microbiology, 83(21), e01366-17. https://doi.org/10.1128/AEM.01366-17

Keller, M., Zengler, K., 2004. Tapping into microbial diversity. Nature Reviews Microbiology, 2(2), 141-150. https://doi.org/10.1038/nrmicro819

Kobayashi, H., 1985. A proton-translocating ATPase regulates $\mathrm{pH}$ of the bacterial cytoplasm. Journal of Biological Chemistry, 260(1), 72-76.

https://www.jbc.org/content/260/1/72.short

Könneke, M., Bernhard, A.E., José, R., Walker, C.B., Waterbury, J.B., Stahl, D.A., 2005. Isolation of an autotrophic ammonia-oxidizing marine archaeon. Nature, 437, 543-546.

https://doi.org/10.1038/nature03911

Köpke, B., Wilms, R., Engelen, B., Cypionka, H., Sass, H., 2005. Microbial diversity in coastal subsurface sediments: a cultivation approach using various electron acceptors and substrate gradients. Applied and Environmental Microbiology, 71(12), 7819-7830. https://doi.org/10.1128/AEM.71.12.7819-7830.2005

Kurm, V., Van Der Putten, W.H., Hol, W.G., 2019. Cultivation-success of rare soil bacteria is not influenced by incubation time and growth medium. PloS One, 14(1), e0210073. https://doi.org/10.1371/journal.pone.0210073

Lagier, J.-C., Dubourg, G., Million, M., Cadoret, F., Bilen, M., Fenollar, F., Levasseur, A., Rolain, J.M., Fournier, P.-E., Raoult, D., 2018. Culturing the human microbiota and culturomics. Nature Reviews Microbiology, 16(9), 540-550. https://doi.org/10.1038/s41579-018-0041-0

Laiz, L., Gonzalez, J., Saiz-Jimenez, C., 2003. Microbial communities in caves: Ecology, physiology, and effects on paleolithic paintings. In: Koestler, R.J., Koestler, V.H. (Eds.), Art, biology, and conservation: Biodeterioration of works of art. The Metropolitan Museum of Art, New York, p. 210-215. 
Laiz, L., Groth, I., Gonzalez, I., Saiz-Jimenez, C., 1999. Microbiological study of the dripping waters in Altamira cave (Santillana del Mar, Spain). Journal of Microbiological Methods, 36(1-2), 129-138.

https://doi.org/10.1016/S0167-7012(99)00018-4

Lee, H., Kim, D.U., Lee, S., Park, S., Yoon, J.H., Seong, C.N., Ka, J.O., 2017. Reyranella terrae sp. nov., isolated from an agricultural soil, and emended description of the genus Reyranella. International Journal of Systematic and Evolutionary Microbiology, 67(6), 2031-2035. https://doi.org/10.1099/ijsem.0.001913

Leo, F. d., Iero, A., Zammit, G., Urzi, C.E., 2012. Chemoorganotrophic bacteria isolated from biodeteriorated surfaces in cave and catacombs. International Journal of Speleology, 41(2), 125-136. https://doi.org/10.5038/1827-806X.41.2.1

Li, L., Mendis, N., Trigui, H., Oliver, J.D., Faucher, S.P., 2014. The importance of the viable but non-culturable state in human bacterial pathogens. Frontiers in Microbiology, 5, 258.

https://doi.org/10.3389/fmicb.2014.00258

Lozupone, C., Knight, R., 2005. UniFrac: a new phylogenetic method for comparing microbial communities. Applied and Environmental Microbiology, 71(12), 8228-8235.

https://doi.org/10.1128/AEM.71.12.8228-8235.2005

Martin, S.E., Flowers, R.S., Ordal, Z.J., 1976. Catalase: its effect on microbial enumeration. Applied and Environmental Microbiology, 32(5), 731-734. https://doi.org/10.1128/AEM.32.5.731-734.1976

McMurdie, P.J., Holmes, S., 2013. phyloseq: an R package for reproducible interactive analysis and graphics of microbiome census data. PloS One, 8(4), e61217. https://doi.org/10.1371/journal.pone.0061217

Nguyen, T.M., Seo, C., Ji, M., Paik, M.-J., Myung, S.W., Kim, J., 2018. Effective soil extraction method for cultivating previously uncultured soil bacteria. Applied and Environmental Microbiology, 84(24), e01145-18. https://doi.org/10.1128/AEM.01145-18

Nichols, D., Cahoon, N., Trakhtenberg, E., Pham, L., Mehta, A., Belanger, A., Kanigan, T., Lewis, K., Epstein, S., 2010. Use of iChip for high-throughput in situ cultivation of "uncultivable" microbial species. Applied and Environmental Microbiology, 76(8), 2445-2450. https://doi.org/10.1128/AEM.01754-09

Oren, A., 2014. The Family Xanthobacteraceae. In: Rosenberg, E., DeLong, E., Lory, S., Stackebrandt, E., Thompson, F. (Eds.), The Prokaryotes - Alphaproteobacteria and Betaproteobacteria. Springer, Berlin, p. 709-726.

Overmann, J., Abt, B., Sikorski, J., 2017. Present and future of culturing bacteria. Annual Review of Microbiology, 71, 711-730.

https://doi.org/10.1146/annurev-micro-090816-093449

Padan, E., Bibi, E., Ito, M., Krulwich, T.A., 2005. Alkaline $\mathrm{pH}$ homeostasis in bacteria: new insights. Biochimica et Biophysica Acta (BBA)-Biomembranes, 1717(2), 6788. https://doi.org/10.1016/j.bbamem.2005.09.010

Pagnier, I., Raoult, D., La Scola, B., 2011. Isolation and characterization of Reyranella massiliensis gen. nov., sp. nov. from freshwater samples by using an amoeba co-culture procedure. International Journal of Systematic and Evolutionary Microbiology, 61(9), 2151-2154. https://doi.org/10.1099/ijs.0.025775-0

Parada, A.E., Needham, D.M., Fuhrman, J.A., 2016. Every base matters: assessing small subunit rRNA primers for marine microbiomes with mock communities, time series and global field samples. Environmental Microbiology, 18(5), 1403-1414.

https://doi.org/10.1111/1462-2920.13023
Pawlowski, A.C., Wang, W., Koteva, K., Barton, H.A., McArthur, A.G., Wright, G.D., 2016. A diverse intrinsic antibiotic resistome from a cave bacterium. Nature Communications, $7,13803$.

https://doi.org/10.1038/ncomms 13803

Quast, C., Pruesse, E., Yilmaz, P., Gerken, J., Schweer, T., Yarza, P., Peplies, J., Glöckner, F.O., 2013. The SILVA ribosomal RNA gene database project: improved data processing and web-based tools. Nucleic Acids Research, 41(D1), D590-D596.

https://doi.org/10.1093/nar/gks 1219

$\mathrm{R}$ Core Team, 2019. R: A Language and Environment for Statistical Computing. R Foundation for Statistical Computing, Vienna, Austria.

Rappé, M.S., Connon, S.A., Vergin, K.L., Giovannoni, S.J., 2002. Cultivation of the ubiquitous SAR11 marine bacterioplankton clade. Nature, 418, 630-633. https://doi.org/10.1038/nature00917

Rideout, J.R., He, Y., Navas-Molina, J.A., Walters, W.A., Ursell, L.K., Gibbons, S.M., Chase, J., McDonald, D., Gonzalez, A., Robbins-Pianka, A., et al., 2014. Subsampled open-reference clustering creates consistent, comprehensive OTU definitions and scales to billions of sequences. PeerJ, 2, e545. https://doi.org/10.7717/peerj.545

Sangwan, P., Kovac, S., Davis, K.E., Sait, M., Janssen, P.H., 2005. Detection and cultivation of soil Verrucomicrobia. Applied and Environmental Microbiology, 71(12), 8402-8410. https://doi.org/10.1128/AEM.71.12.8402-8410.2005 Shirling, E.T., Gottlieb, D., 1966. Methods for characterization of Streptomyces species. International Journal of Systematic Bacteriology, 16(3), 313-340. https://doi.org/10.1099/00207713-16-3-313

Simpson, E.H., 1949. Measurement of diversity. Nature, 163, 688. https://doi.org/10.1038/163688a0

Solden, L., Lloyd, K., Wrighton, K., 2016. The bright side of microbial dark matter: lessons learned from the uncultivated majority. Current Opinion in Microbiology, 31, 217-226.

https://doi.org/10.1016/j.mib.2016.04.020

Song, J., Oh, H.-M., Cho, J.-C., 2009. Improved culturability of SAR11 strains in dilution-to-extinction culturing from the East Sea, West Pacific Ocean. FEMS Microbiology Letters, 295(2), 141-147. https://doi.org/10.1111/j.1574-6968.2009.01623.x

Staley, J.T., Konopka, A., 1985. Measurement of in situ activities of nonphotosynthetic microorganisms in aquatic and terrestrial habitats. Annual Review of Microbiology, 39(1), 321-346.

https://doi.org/10.1146/annurev.mi.39.100185.001541

Stewart, E.J., 2012. Growing unculturable bacteria. Journal of Bacteriology, 194(16), 4151-4160. https://doi.org/10.1128/JB.00345-12

Tanaka, T., Kawasaki, K., Daimon, S., Kitagawa, W., Yamamoto, K., Tamaki, H., Tanaka, M., Nakatsu, C.H., Kamagata, Y., 2014. A hidden pitfall in the preparation of agar media undermines microorganism cultivability. Applied and Environmental Microbiology, 80(24), 76597666. https://doi.org/10.1128/AEM.02741-14

Teixeira, L., Merquior, V., 2014. The Family Moraxellaceae. In: Rosenberg, E., DeLong, E., Lory, S., Stackebrandt, E., Thompson, F. (Eds.), The Prokaryotes - Gammaproteobacteria. Springer, Berlin, p. 443-476. https://doi.org/10.1007/978-3-642-38922-1 245

Tiwari, K., Gupta, R.K., 2013. Diversity and isolation of rare actinomycetes: an overview. Critical Reviews in Microbiology, 39(3), 256-294.

https://doi.org/10.3109/1040841X.2012.709819 
Tripp, H.J., Kitner, J.B., Schwalbach, M.S., Dacey, J.W., Wilhelm, L.J., Giovannoni, S.J., 2008. SAR11 marine bacteria require exogenous reduced sulphur for growth. Nature, 452, 741-744.

https://doi.org/10.1038/nature06776

Vartoukian, S.R., Palmer, R.M., Wade, W.G., 2010. Strategies for culture of "unculturable" bacteria. FEMS Microbiology Letters, 309(1), 1-7. https://doi.org/10.1111/j.1574-6968.2010.02000.x

Watve, M., Shejval, V., Sonawane, C., Rahalkar, M., Matapurkar, A., Shouche, Y., Patole, M., Phadnis, N., Champhenkar, A., Damle, K., et al., 2000. The ' $\mathrm{K}$ ' selected oligophilic bacteria: A key to uncultured diversity? Current Science, 78(12), 1535-1542. https://www.jstor.org/stable/24104330
West Virginia Cave Conservancy, 2018. Management Plan for the Preserve for the New Entrance to Maxwelton Sink Cave.

https://wvcc.net/maxwelton-management-plan

Wickham, H., 2016. ggplot2: Elegant graphics for data analysis. Springer, Cham, $260 \mathrm{p}$.

https://doi.org/10.1007/978-3-319-24277-4

Winterberg, H., 1898. Zur methodik der bakterienzählung. Medical Microbiology and Immunology, 29(1), 75-93. https://doi.org/10.1007/BF02217377

Zengler, K., 2009. Central role of the cell in microbial ecology. Microbiology and Molecular Biology Reviews, 73(4), 712-729.

https://doi.org/10.1128/MMBR.00027-09 